\title{
Situación epidemiológica del cáncer de próstata en España
}

\author{
Granado de la Orden S*, Saá Requejo C**, Quintás Viqueira A***. \\ *Servicio de Medicina Preventiva y Gestión de Calidad. Hospital General Universitario Gregorio Marañón. Madrid. \\ ${ }^{* *}$ Servicio de Medicina Preventiva. Hospital Ramón y Cajal. Madrid. \\ ***Servicio de Medicina Preventiva. Hospital Universitario de Guadalajara. Guadalajara.
}

Actas Urol Esp 2006; 30 (6): 574-582

\section{RESUMEN \\ SITUACIÓN EPIDEMIOLÓGICA DEL CÁNCER DE PRÓSTATA EN ESPAÑA.}

El cáncer de próstata es el tercer tumor más frecuente en varones españoles y la tercera causa de muerte por cáncer. Su incidencia aumenta con la edad. Un 90\% de casos se diagnostican en mayores de 65 años. La etiología es poco conocida relacionándose con exposiciones ambientales, estilos de vida, antecedentes familiares y factores genéticos. En el año 2002 la tasa de mortalidad (situada entre las más bajas de Europa) fue 21,5 casos por 100.000 habitantes, superándose las 5.000 defunciones. La tendencia de mortalidad ha ido en aumento hasta 1998, año en el que empieza a disminuir en relación con mejoras en el diagnóstico y tratamiento. Al estudiar la incidencia del cáncer de próstata nos encontramos con la dificultad debida a la escasez de registros poblacionales de cáncer. Se han realizado estimaciones a partir de los datos disponibles, detectándose una tasa de incidencia de 45,33 por 100.000 habitantes, también entre las más bajas de Europa. La incidencia anual de cáncer de próstata ha aumentado en todos los registros españoles debido a mejoras en los mismos, pero también al desarrollo de las pruebas diagnósticas, que han condicionado una mejor supervivencia desde principios de los 90 (86\% al año del diagnóstico y $65,5 \%$ a los cinco años) comparable a la de otros países de nuestro entorno. Es necesario ampliar el sistema de registro de cáncer para conocer la incidencia y la prevalencia, evaluar la supervivencia y la eficacia de los programas de detección precoz y mejorar en el conocimiento de los factores de riesgo.

Palabras clave: Cáncer de próstata. Mortalidad. Incidencia. Supervivencia. Prevalencia.

\section{ABSTRACT \\ EPIDEMIOLOGICAL SITUATION OF PROSTATE CANCER IN SPAIN.}

Prostate cancer is the third most frequent neoplasms in Spanish men and the third cause of cancer death. Incidence grows up with the increase of age. $90 \%$ of cases are diagnostic in people over 65 years old. Etiology is quite unknown and has been associated with environmental exposure, life style, family sign and genetic factors. In 2002 mortality rate was 21.5/ 100.000 (situated among the lowest in Europe), with more than 5.000 deaths. Trend of mortality has grown up until 1998, from this year it has decreased due to improve on diagnostic and treatment. In order to study prostate cancer incidence we find a difficulty due to shortage of population cancer register. Estimations have found incidence rates of 45.33/100.000 which are among the lowest in Europe. Annual incidence of prostatic cancer has grown up in all Spanish registers, not only improve in register systems explains it, but also the development of diagnosis tests with a higher survival from the beginning of $90^{\mathrm{s}}$ (86\% the first year after diagnosis and 65,5\% five years after diagnosis), similar to other European countries. Blow up the cancer register system is necessary to know the incidence and prevalence, to assess survival and effectiveness of screening programs and to improve the knowledge of risk factors.

Keywords: Prostate cancer. Mortality. Incidence. Survival. Prevalence. 
$\mathrm{E}_{\mathrm{s}}^{1}$ cáncer constituye uno de los problemas sanitarios más importantes en nuestro medio $^{1}$, tanto en términos de morbimortalidad como de impacto social, económico o sobre la calidad de vida, afectando a una de cada tres personas a lo largo de su vida ${ }^{2}$

En el año 2000 el cáncer pasó a ser, para el conjunto de grupos de edad, la primera causa de muerte en España en varones y la segunda en mujeres, después de las enfermedades cardiovasculares, registrándose 91.623 muertes (57.382 en varones y 34.241 en mujeres), lo que supuso el $25,6 \%$ de todas las defunciones ${ }^{3}$

El cáncer de próstata es la tercera localización tumoral más frecuente en varones españoles (excluyendo los tumores de piel no melanoma), y la tercera causa de muerte por cáncer, en ambos casos por detrás del cáncer de pulmón y el colorrectal.

Se estima que uno de cada seis varones desarrollará cáncer de próstata a lo largo de su vida ${ }^{4}$. La probabilidad de desarrollar un cáncer de próstata aumenta con la edad, de forma que nueve de cada diez casos aparecen en mayores de 65 años. Anatomopatológicamente, más del 95\% son adenocarcinomas, mientras que los restantes son carcinomas de células escamosas o transicionales y sarcomas ${ }^{5}$.

La etiología y los agentes que condicionan la evolución hacia un tumor clínicamente manifiesto son poco conocidos, si bien se considera un proceso multifactorial relacionado con ciertas exposiciones ambientales, estilos de vida, antecedentes familiares de cáncer de próstata y factores genéticos. Estudios recientes señalan la importancia de la base genética de la enfermedad, que condiciona tanto la susceptibilidad a la misma como la agresividad clínica ${ }^{6}$. La edad avanzada, la historia familiar de cáncer de próstata y los orígenes africanos son factores de riesgo conocidos. El efecto de la actividad física, la actividad sexual, la exposición al cadmio, el hábito tabáquico, el consumo de alcohol, la dieta rica en grasas saturadas de origen animal y el consumo de carne roja sobre la aparición de cáncer de próstata es actualmente controvertido. Mientras que algunos estudios han propuesto que el consumo de Zinc podría favorecer la aparición del cáncer (a través de un aumento en los niveles de testoste- rona), otros le han otorgado un papel protector, que también se ha atribuido a la dieta rica en vitamina $\mathrm{E}$, selenio y vitamina $\mathrm{D}^{7,8}$.

El objetivo de este trabajo es revisar la situación epidemiológica actual del cáncer de próstata en España atendiendo a cuatro indicadores: mortalidad, incidencia, supervivencia y prevalencia.

Mortalidad por cáncer de próstata en España y situación respecto a otros países de la Unión Europea

El cáncer de próstata es el tumor maligno que presenta la tercera tasa más alta de mortalidad en hombres en España, tras el cáncer de pulmón y el colorrectal, superando las 5.000 muertes al año. Según datos del Centro Nacional de Epidemiología, en el año 2002 el cáncer de próstata fue responsable de 5.664 defunciones, con una tasa cruda de mortalidad de 29,31 por 100.000 habitantes y una tasa ajustada (población estándar europea) de 21,48 casos por 100.000 habitantes. El porcentaje de cambio anual en el periodo comprendido entre los años 1991 y 2000 fue del 0,13\% (IC 95\%: -0,17-0,43). La edad media de los fallecidos era de 74,93 años. Es un tumor en el que se observa claramente el efecto de la edad sobre la mortalidad: el cáncer de próstata es raro en hombres menores de 50 años, pero luego su incidencia aumenta rápidamente con la edad, de forma que el 90\% de los casos aparecen en mayores de 65 años y la muerte se produce alrededor de los 75 años. Este hecho se manifiesta claramente en la tasa truncada que es de 4,24 por 100.000 en el año 2002.

En un estudio realizado por López-Abente ${ }^{9}$ y colaboradores, en el que se investiga la tendencia de la mortalidad por cáncer de próstata en España entre los años 1952 y 1996 desde la perspectiva de las generaciones o cohortes de nacimiento, se observa que la tendencia de mortalidad por este tumor ha sido de un incremento neto cercano al $1 \%$ anual, que disminuye discretamente cuando se restringe el análisis al periodo 1982-1996. Las tasas específicas de mortalidad por edad son ligeramente más altas para las cohortes más recientes, si bien en los más jóvenes esta tendencia no está tan clara. A partir de 1970, la tendencia de la mortalidad según el efecto periodo se mantiene relativamente estable, lo que se podría explicar por las mejoras diagnósticas 
y terapéuticas, ya que la incidencia de este tumor en España entre los años 1973 y 1990 se incrementó en más de un 2\%. Desde 1998 la mortalidad empieza a disminuir (Porcentaje de Cambio Anual 1998-2000: -0,17), disminución que afecta a las Comunidades Autónomas de Baleares, Valencia, Cataluña y Madrid, mientras que en las restantes Comunidades Autónomas la tendencia continua siendo ascendente. Aunque, como se ha señalado anteriormente, los avances en el diagnóstico y tratamiento de este tumor pueden suponer una estabilización de las cifras de mortalidad a pesar del aumento de la incidencia, también la mayor exactitud diagnóstica y de la certificación de la causa de muerte puede condicionar un aumento de la mortalidad.

Las diferencias en la mortalidad por cáncer de próstata entre las provincias españolas no son demasiado pronunciadas y no se aprecia un claro patrón geográfico. Las tasas ajustadas de mortalidad oscilan entre cifras de $15,5-16$ por 100.000 habitantes en las provincias con menor mortalidad (Melilla, Guadalajara, Almería y Cáceres) y cifras de 27,528 por 100.000 en las que presentan mayor mortalidad (Baleares, Las Palmas de Gran Canaria y Castellón) (Figs. 1 y 2).

Según el último informe de EUROSTAT $^{10}$, el cáncer de próstata es la causa del 3\% de todas las muertes de los hombres en la Unión Europea, y el $10 \%$ de los varones que fallecen por tumores malignos lo hacen como consecuencia de este tumor. españolas en 2002.
Se han encontrado diferencias en la mortalidad por cáncer de próstata entre los países del norte y del sur de Europa. Los países escandinavos, Bélgica, Países Bajos y ciertas regiones de Francia presentan alta mortalidad mientras que Bulgaria, Hungría y Rumanía así como los países mediterráneos tienen una mortalidad inferior a la media europea. Las tasas de mortalidad en España se encuentran entre las más bajas de Europa junto con Italia y Grecia.

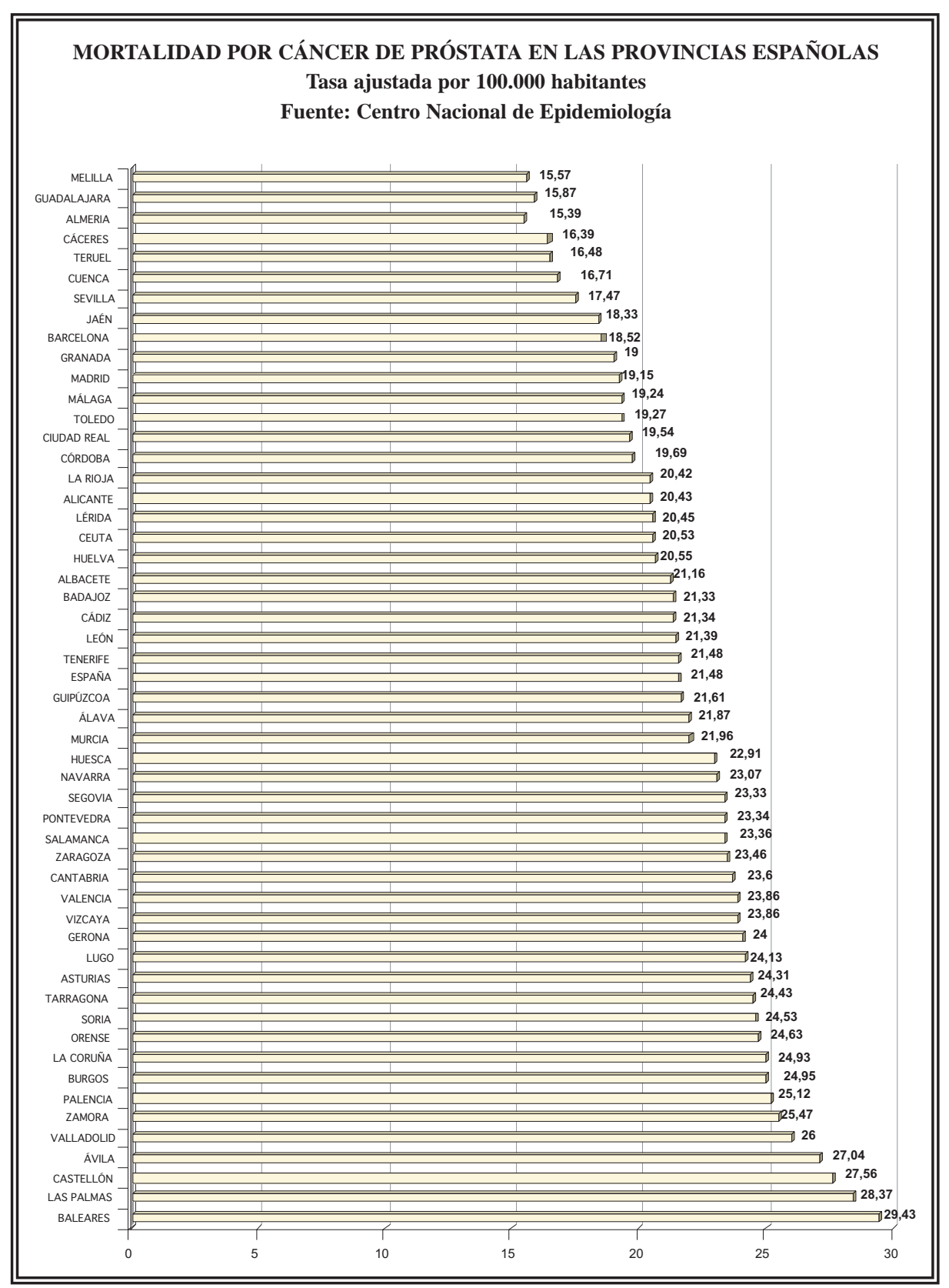

FIGURA 1. Tasas ajustadas de mortalidad por cáncer de próstata en las provincias 


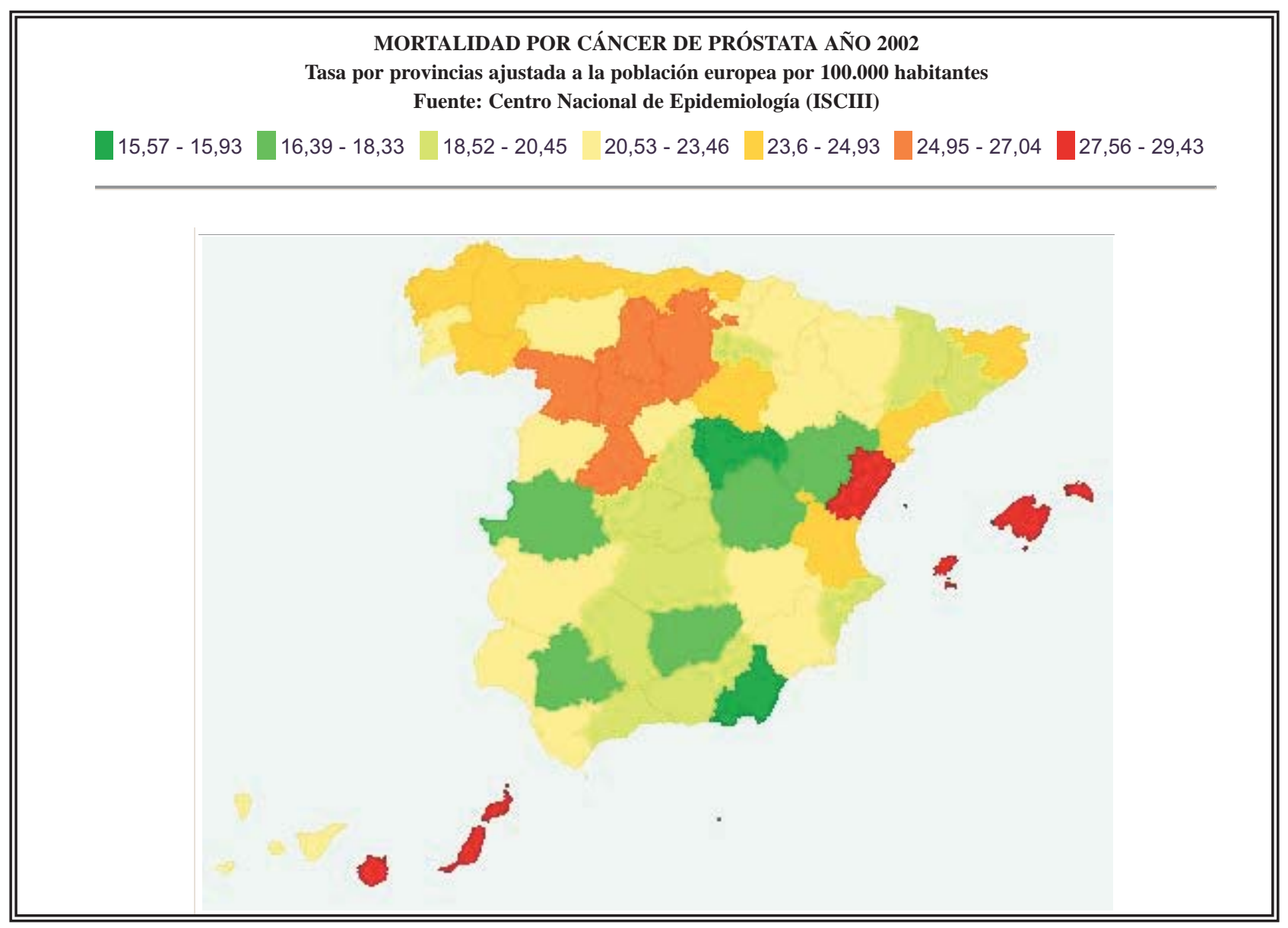

FIGURA 2. Distribución geográfica de la mortalidad por cáncer de próstata en España.

Incidencia del cáncer de próstata en España y situación respecto a otros países europeos (Fig. 3)

La incidencia de cáncer de próstata en una población depende de factores de riesgo no modificables (edad y raza) pero también de factores modificables (utilización libre o restringida del PSA como marcador tumoral, criterios para la indicación de biopsia glandular y agresividad de la técnica de biopsia) que pueden explicar parcialmente las diferencias encontradas en los registros.

La incidencia de cáncer de próstata en España se encuentra, junto a Grecia, entre las más bajas de la Unión Europea. Se ha estimado una incidencia anual de cáncer de próstata en España en el periodo 1997-2000 de 13.212 casos nuevos al año (IC 95\%: 6.245-24.524), con una tasa ajustada de 56,29 por 100.000 habitantes (población estándar europea ${ }^{11}$ ). La tasa de incidencia de cáncer de próstata estimada por la EUCAN para
1998 en España es de 45,33 por 100.000 habitantes ${ }^{17}$ (población estándar europea) con 10.659 casos nuevos en ese año, mientras que la tasa de incidencia estimada en la Unión Europea para ese mismo año es de 67,55 por 100.000 habitantes, más de veinte puntos superior a la española.

En un trabajo realizado por Herranz et $\mathrm{al}^{12}$ en el año 2000, se encontró una incidencia bruta de cáncer de próstata en la Comunidad de Madrid de 100,4 casos por 100.000 varones y una incidencia específica en hombres mayores de 50 años de 367,2 casos por 100.000 y de 550,6 casos por 100.000 en mayores de 80 años.

Se ha observado un incremento en la incidencia de cáncer de próstata en todos los registros españoles. Esto es, en parte, debido a la mejora progresiva de la calidad de la información que contienen. Sin embargo, la incidencia de este tumor ha aumentado en los últimos años debido a tres hechos: el aumento de la esperanza de vida 


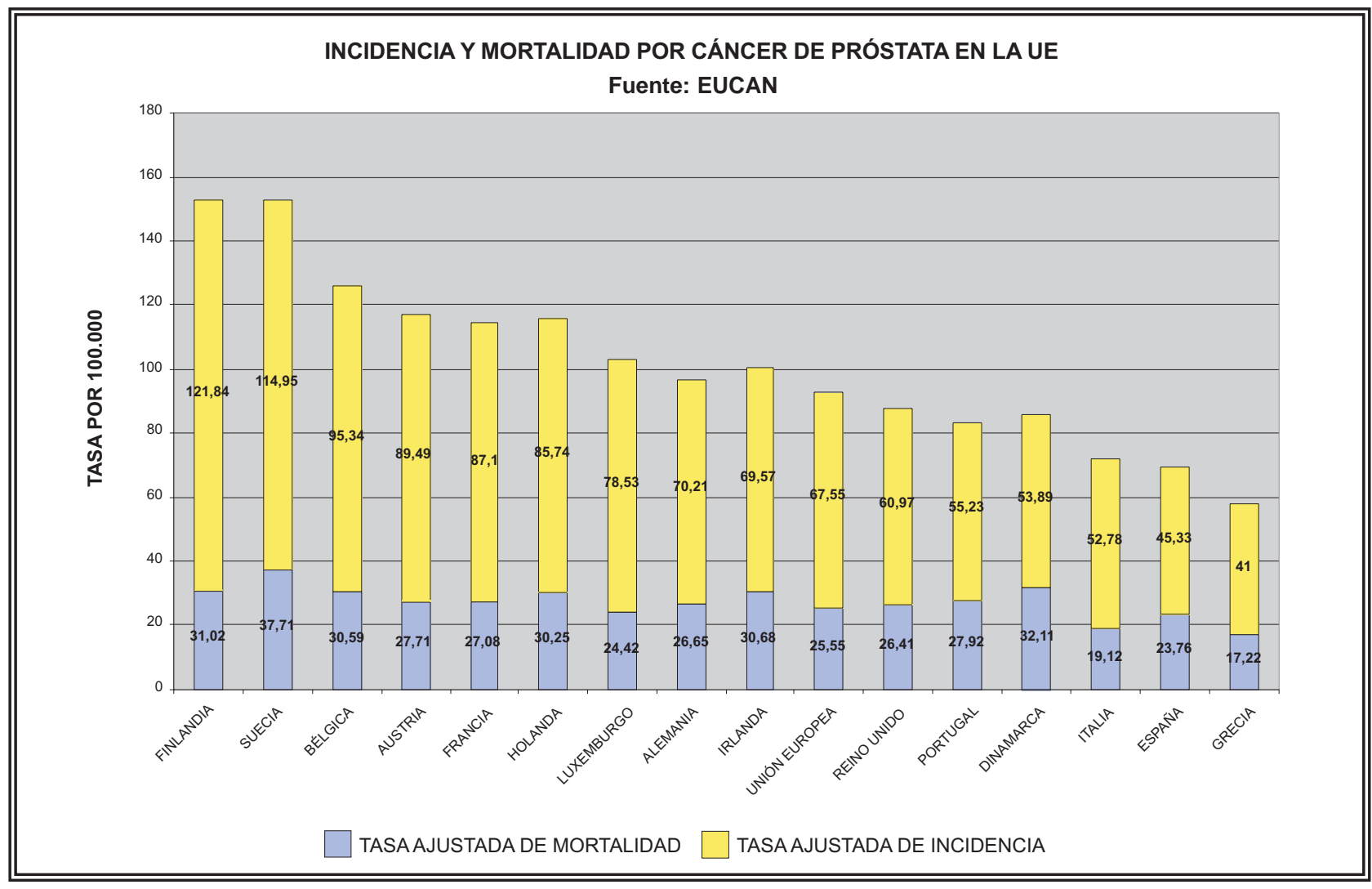

FIGURA 3. Mortalidad por cáncer de próstata en los paises de la Unión Europea.

(con el consiguiente aumento de la edad poblacional), la utilización, desde finales de los años 80, del antígeno prostático específico (PSA) que permite el diagnóstico en fase preclínica aumentando así el número de casos diagnosticados, y la mejora y extensión de los métodos diagnósticos de imagen (Fig. 4).
Los pocos datos existentes parecen apuntar que en el resto de Europa nos encontramos, también, en un periodo de incremento de la incidencia. Así, Murphy et al. encontraron un incremento del $30 \%$ en la incidencia de cáncer de próstata en la región de Yorkshire en el periodo 1981$1990^{13}$, Brewster et al. encontraron en Escocia un

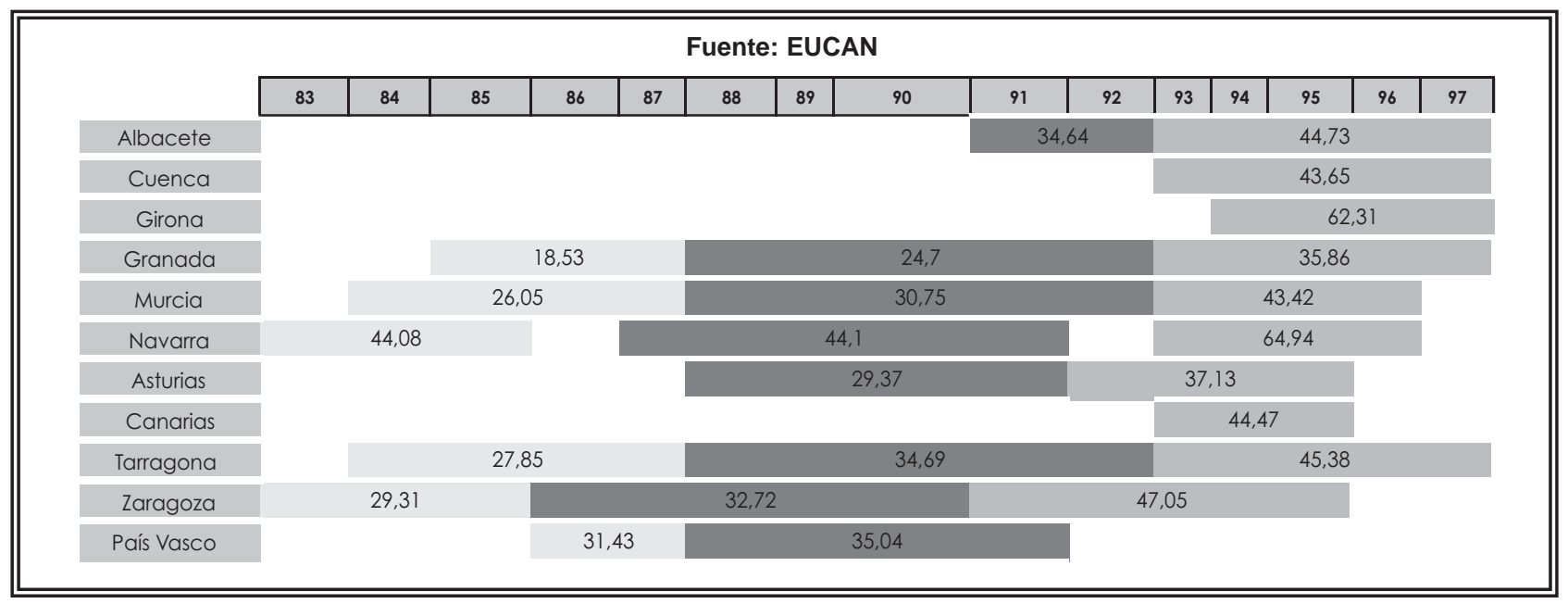

FIGURA 4. Incidencia de cáncer de próstata en los registros poblaciones españoles. 
aumento de la incidencia del 59\% entre los años 1982-1996 ${ }^{14}$ y el registro de tumores de Rótterdam la incidencia aumentó de 62 casos por 100.000 hombres en 1989 a 125 casos por 100.000 en $1995^{15}$ (Fig. 5).

Supervivencia de los pacientes con cáncer de próstata en España y en Europa

Para conocer la supervivencia de los pacientes con cáncer en Europa se han desarrollado los proyectos EUROCARE-1, 2 y 3 que han supuesto el seguimiento de todos los casos, incluidos en los registros poblacionales de cáncer, de los países europeos participantes e informan de la supervivencia global de los pacientes oncológicos. El estudio EUROCARE-3 analiza la supervivencia de 1.815.584 pacientes adultos diagnosticados de cáncer entre los años 1990 y 1994 en 22 países europeos. Los Registros poblacionales españoles que han participado en este proyecto son los de País Vasco, Navarra, Tarragona, Mallorca, Murcia y Granada que aportan 3.635 pacientes.

Según EUROCARE-3 la supervivencia de los pacientes diagnosticados de cáncer de próstata en España es del 86\% al año del diagnóstico (IC 95\%: $84,7-87,4)$ y del $65,5 \%$ a los 5 años (IC 95\%: 63$68)^{16}$. Esta supervivencia ha mejorado respecto a los datos aportados por el estudio EUROCARE-2 que determinaba una supervivencia para los pacientes diagnosticados de cáncer de próstata en España entre los años 1985-1989 del 83\% al año del diagnóstico y el 54\% a los cinco años (IC 95\%: 51-58) ${ }^{17}$. La determinación del Antígeno Prostático Específico desde principios de los años 90 y el hallazgo casual de tumores prostáticos en el análisis anatomopatológico de tejidos procedentes de resecciones transuretrales realizadas a pacientes diagnosticados de hipertrofia benigna de próstata (en el estudio realizado por Herranz et al. ${ }^{4}$ hasta un $12 \%$ de los cánceres de próstata se diagnosticaron de forma incidental debido a cirugía prostática y en un estudio realizado en el Área 8 de la Comunidad de Madrid $^{18}$ se encontraron un 36,9\% de cánceres prostáticos incidentales) han ocasionado, por una parte, un aumento de la incidencia de cáncer de próstata, pero también una mejora de la supervivencia al tratarse de tumores localizados y con mejor pronóstico ${ }^{19}$.
La supervivencia de los pacientes con cáncer de próstata en España es comparable a la de otros países de nuestro entorno. EUROCARE-3 señala una supervivencia media en Europa al año del diagnóstico de 87,8\% (IC 95\%: 87,3-88,3) y de 65,4\% (IC 95\%: 64,4-66,4) a los cinco años. Austria, Francia y Alemania presentan las mejores supervivencias (iguales o superiores al $75 \%$ a los cinco años) mientras que Dinamarca, Reino Unido y Portugal presentan las supervivencias más bajas (inferiores al 55\%). Sin embargo, es necesario tener en cuenta que la baja supervivencia en países como Dinamarca o Reino Unido se relaciona con niveles bajos de incidencia, mientras que se observan mejores supervivencias en países que presentan mayores tasas de incidencia como Francia, Alemania, Austria o Países Bajos. En regiones como Tirol en Austria, donde la supervivencia a los cinco años es superior al $80 \%$, la utilización de la prueba del Antígeno Prostático Específico está ampliamente extendida desde $1992^{20,21}$. España se sitúa entre los países con baja incidencia y mortalidad y una supervivencia próxima a la media europea (Fig. 6).

Prevalencia del cáncer de próstata en España

La International Agency for Research on Cancer (IARC) ha realizado, a partir de los datos de incidencia y supervivencia, una estimación del número de casos prevalentes de cáncer de próstata en España para el año 1998. De esta manera, se ha estimado una prevalencia parcial correspondiente a pacientes diagnosticados en el último año de 9.430 casos y de pacientes diagnosticados en los últimos cinco años de 36.492 casos $^{17}$. El cáncer de próstata es uno de los más prevalentes en varones españoles junto con los tumores de vejiga, colorrectal y pulmón, tanto a uno como a cinco años suponiendo el $15,48 \%$ de todos los tumores a un año y el $15,2 \%$ a cinco años ${ }^{17}$. Es previsible que la prevalencia de este tumor continúe aumentando en los próximos años como consecuencia de la cada vez mayor utilización de técnicas de diagnóstico precoz, que permiten la detección de tumores que de otra forma podrían no haber sido nunca detectados, la mejor supervivencia de estos tumores diagnosticados en fases precoces y el aumento de la longevidad, hecho que se asocia claramente a la aparición de este cáncer. 


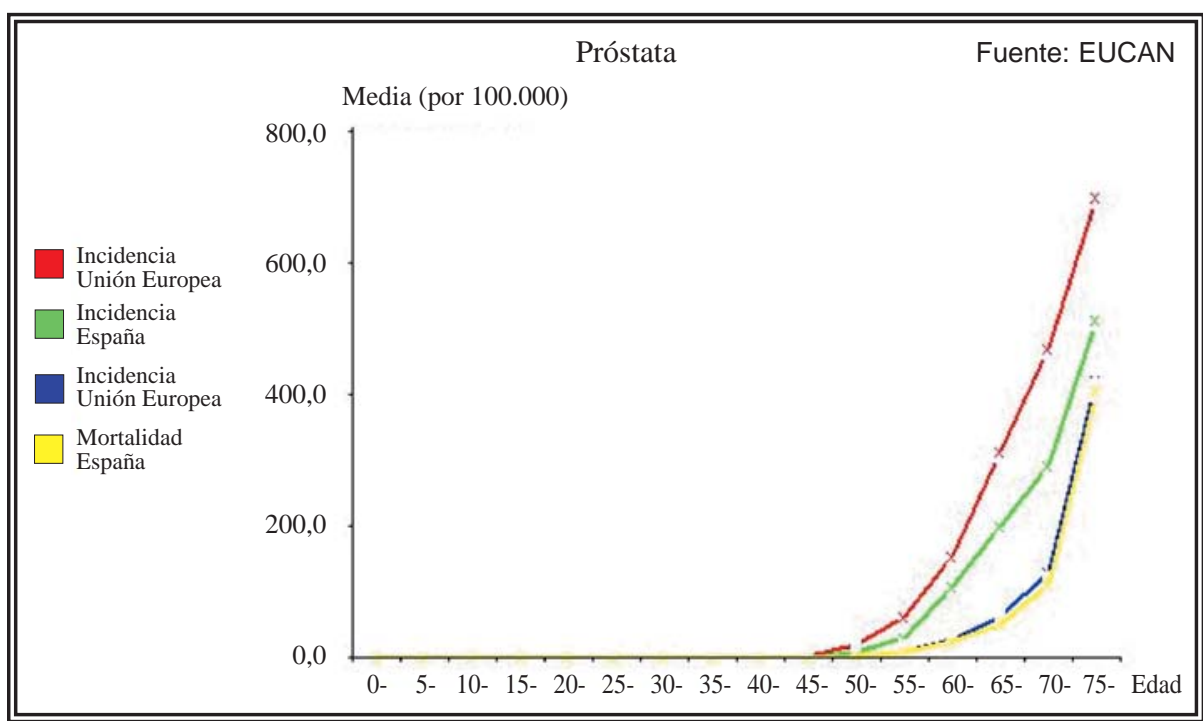

FIGURA 5. Incidencia y mortalidad por cáncer de próstata en España y los paises de la Unión Europea.

\section{DISCUSIÓN}

El cáncer de próstata es uno de los tumores más frecuentes en el sexo masculino. Su incidencia aumenta debido a la mayor esperanza de vida y al desarrollo de técnicas diagnósticas que permiten la detección de tumores asintomáticos que años atrás pasaban desapercibidos. Es esperable que en los próximos años, con el envejecimiento progresivo de la población, esta incidencia continúe aumentando. Esto lo convierte en un impor- tante problema sanitario debido a los cuidados que precisan los pacientes oncológicos que además en este caso asocian otro problema que es la avanzada edad, por lo que es frecuente que el cáncer coexista junto a otras patologías. En el trabajo realizado por F. Herranz y colaboradores $^{4}$ en el que se analizan los datos de 1.745 pacientes diagnosticados de cáncer de próstata en la Comunidad de Madrid, el $67 \%$ de los evaluados presentaban patología asociada de distinta severidad y un $36 \%$ patología múltiple, lo que puede generar mayores complicaciones $\mathrm{y}$ dificultades terapéuticas.

La evolución de la incidencia del cáncer de próstata en España debe ser objeto de estudios específicos. Actualmente los datos disponibles se obtienen a partir de estimaciones realizadas con los datos de los registros poblacionales existentes, por lo que no se tienen en cuenta las posibles variaciones geográficas y se desconoce la incidencia real.

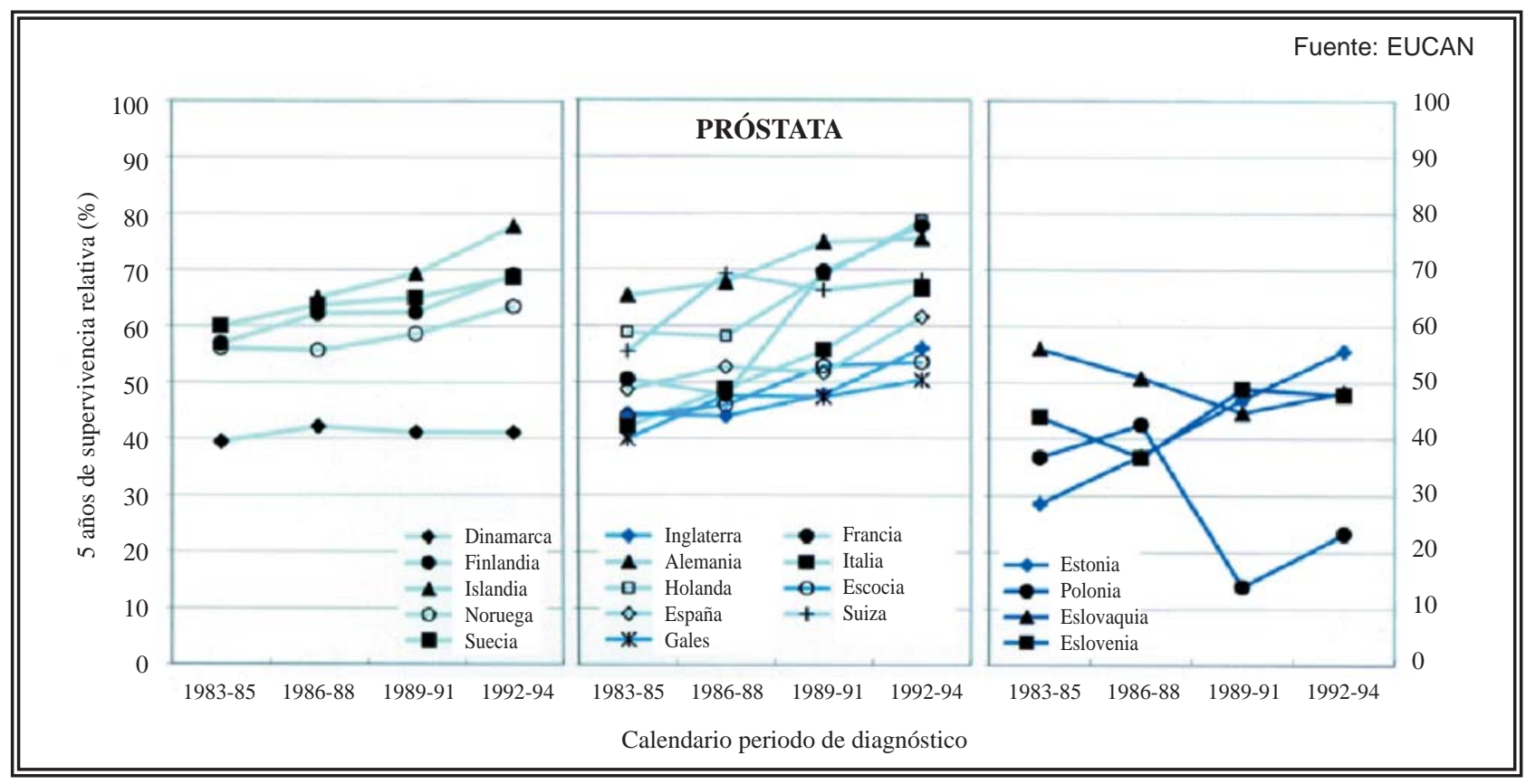

FIGURA 6. Evolución de la supervivencia a 5 años en los paises de la Unión Europea. 
La mortalidad en España se mantiene estable, si bien hay algunas diferencias geográficas que no son significativas. La causa de esta estabilidad, a pesar del aumento de la incidencia, es el diagnóstico precoz y las mejoras terapéuticas. La utilización de la prueba de detección del Antígeno Prostático Específico, cada vez más extendida, si bien cuestionada y no utilizada como técnica de despistaje poblacional, se ha asociado en algunas series a un aumento de la supervivencia. Es necesario continuar investigando para encontrar una técnica sensible que pueda ser utilizada a nivel poblacional pero lo suficientemente específica como para que el número de falsos positivos, que obligan a la realización de técnicas invasivas, sea lo menor posible.

La causas del cáncer de próstata así como los factores que condicionan su evolución clínica permanecen todavía bastante desconocidos. Estudios recientes apuntan la gran importancia de la base genética de este tumor. Sin embargo dadas las diferencias geográficas existentes, observadas por ejemplo entre países de la Unión Europea, es posible que factores ambientales asî como estilos de vida también estén implicados. El problema es que de momento no son suficientemente conocidos como para poder diseñar estrategias preventivas que sean eficaces. Es necesario continuar investigando en este campo para que en un futuro seamos capaces de prevenir la aparición de este tumor y podamos detectar grupos de riesgo que puedan beneficiarse de la aplicación de técnicas de diagnóstico precoz.

La incidencia y la mortalidad por cáncer de próstata en España se encuentran entre las más bajas de Europa. Sin embargo, es necesario prestar atención a la calidad de los registros de donde obtenemos los datos, puesto que se necesita que sean homogéneos para poder establecer comparaciones.

Así, la información sobre mortalidad, único indicador para cáncer con carácter universal en nuestro país, se obtiene de los certificados de defunción, por lo que la fiabilidad de esta información depende de la calidad de los mismos. Puede suceder que se atribuyan muertes a causas subyacentes o secundarias al tumor, infravalorándose las cifras de mortalidad por cáncer. Es necesario promover en todas las Comunidades
Autónomas sistemas y programas para mantener y mejorar los certificados de defunción, dado que es la mejor fuente de información sobre mortalidad actualmente disponible.

Los registros poblacionales de cáncer, existentes actualmente sólo en algunas Comunidades Autónomas y provincias, son necesarios para obtener información sobre la incidencia y la prevalencia del cáncer, evaluar la supervivencia de los pacientes y los programas de diagnóstico precoz establecidos e investigar los factores de riesgo. Debemos insistir, por tanto, en la creación de registros poblacionales en aquellas Comunidades Autónomas que carecen de ellos y mejorar la calidad de la información de los ya existentes. Es necesario que la recogida de información en los diferentes registros siga unas pautas comunes con el fin de que exista una homogeneidad que nos permita establecer comparaciones.

Por último señalar que la estrategia para luchar contra el cáncer de próstata es labor de diferentes disciplinas. La investigación en el campo de la urología y la genética es imprescindible para avanzar en el conocimiento de las causas de la enfermedad. Es también necesario el desarrollo de técnicas de diagnóstico precoz fiables y aplicables a amplios sectores de población que nos permitan detectar tumores en estadios iniciales fácilmente tratables. El conocimiento de las características epidemiológicas de la enfermedad nos ayuda a conseguir los objetivos anteriores y a diseñar estrategias de investigación e intervención futuras.

Agradecimientos: A los Drs. Beatriz Pérez del Centro Nacional de Epidemiología, Felipe Herranz, Dolores Vigil y Paz Rodríguez del H.G.U. Gregorio Marañón.

\section{REFERENCIAS}

1. Brown ML, Lipscomb J, Snyder C. The burden of cancer: economic cost and quality of life. Annu Rev Public Health 2001;22:91-113.

2. Moreno V, Sánchez V, Galceran J, Borrás JM, Bosch X. Riesgo de enfermar y morir por cáncer en Cataluña. Med Clin (Barc), 1998; 110:86-93.

3. Ministerio de Sanidad y Consumo. Área de Epidemiología Ambiental y Cáncer. Centro Nacional de Epidemiología. Instituto de Salud Carlos III. Plan integral del cáncer. Situación del cáncer en España.

4. Herranz F, Arias F, Arrizabalaga M, Calahorra FJ, Carballido J, Diz R et al. El cáncer de próstata en la Comunidad de Madrid en el año 2000. II- Presentación y diagnóstico. Actas Urol Esp. 2003;27(5):335-344. 
5. Fernández A, Gil J, Otero G. Epidemiología del cáncer de próstata. Epidemiología descriptiva y analítica en oncología urológica. Actas Urol Esp.1998:43-66.

6. Schaid D. The complex genetic epidemiology of prostate cancer. Human Molecular Genetics 2004, Vol. 13, Review Issue 1 .

7. Bostwick DG, Burke HB, Djakiew D, Euling S, Ho SM, Landolph J, et al. Human prostate cancer risk factors. Cancer 2004 15;101 (10 Suppl): 2371-2490.

8. Boyle P, Severi G, Giles GG. The epidemiology of prostate cancer. Urol Clin North Am. 2003;30(2):209-217.

9. López-Abente G, Pollán M, Aragonés N, Pérez B, Lláer A, Pérez J, et al. Tendencias de la mortalidad en España, 1952-1996. Efecto de la edad, de la cohorte de nacimiento y del periodo de muerte: Instituto de Salud Carlos III. Madrid 2002.

10. Regions: Statistical yearbook 2004 de la UE. Informe elaborado por la Oficina para las Publicaciones Oficiales de las Comunidades Europeas en el año 2004.

11. Centro Nacional de Epidemiología. Estimación del número de casos anuales de cáncer de próstata y tasa ajustada para España realizada a partir de los datos de incidencia y mortalidad de las provincias con registros poblacionales de cáncer. 2003.

12. Herranz Amo, F, Arias Fúnez, F, Arrizabalaga Moreno M, Calahorra Fernández FJ, Carballido Rodríguez J, et al. El cáncer de próstata en la Comunidad de Madrid en el año 2000: -Incidencia. Actas Urol Esp. 2003;27(5):323-334.

13. Murphy M, Johston C, Whelan P, Rider L, Lloyd SN. Changing trends in prostatic cancer. BJU International 1999;83(7):786-791.

14. Brewster DH, Fraser LA, Harris V, Black RJ. Rising incidence of prostate cancer in Scotland: increased risk or increased detection?. BJU International 2000;85(4):463473.

15. Spapen SJJC, Damhuis RAM, Kirkels WJ.:Trends in the curative treatment of localized prostate cancer after the introduction of prostate-specific antigen: data from the Rotterdam cancer registry. BJU International 2000;85(4): 474-480.

16. Sant M, Aareleid T, Berrino F, Bielska Lasata M, Carli PM, Faivre $J$ and the European Working Group. Eurocare-3: survival of cancer patients diagnosed 1990-94, results and commentary. Annals of Oncology; 2003;14(Supplement 5): v61-v118.

17. Berrino F, Capoccacia R, Estève J, Gatta G, Hakulinen T, Micheli A et al. Survival of cancer patients in Europe: the EUROCARE-2 Study. IARC Scientific Publications No. 151. Lyon 1999.

18. Arrizabalaga Moreno M, García González JI, Díez Rodríguez JM, et al. Indicadores epidemiológicos del adenocarcinoma de próstata. Resultados sobre 436 pacientes. Actas Urol Esp. 1997;21:852-861.

19. Evans HS, Moller H. Recent trends in prostate cancer incidence and mortality in southeast England. Eur Urol. 2003; 43(4):337-341.
20. Micheli A, Coebergh JW, Mugno E and the EUROCARE Working group. European health systems and cancer care. Ann Oncol. 2003;14(Suppl 5):v41-v60.

21. Horninger W, Reissigl A, Rogatsch H. Prostate cancer screening in Tyrol, Austria: experience and results. Eur Urol 1999;35(5-6):523-538.

22. Luján Galan M, Paez BordaA, Llanes L Gómez de Vicente JM,Romero Cajigal I et al.Rasgos diferenciales del cancer de prostata procedente de un programa de detección pre$\mathrm{coz}$ frente al detectado en una consulta de urología. Actas Urol Esp. 2000;24(8):640-643,.

23. Van der Cruijsen-Koeter IW, Vis AN, Roobol MJ, Wildhagen MF, de Koning $\mathrm{HJ}$, van der Kwast TH, Schroder FH. Comparison of screen detected and clinically diagnosed prostate cancer in the European randomized study of screening for prostate cancer, section Rotterdam. J Urol. 2005; 174(1):121-125.

24. Páez Borda A, Luján Galán M, Llanes González L, Romero Cagigal I, de la Cal López MA, Miravalles González E et al A. Análisis de la utilización del antígeno específico prostático (PSA) en un área sanitaria de la Comunidad de Madrid. Actas Urol Esp. 2001;25(10):698-703, .

25. Caballero Alcántara JE, Marchal Escalona C, Padilla León M, Rodrigo Fernández I Aumento de la tasa cruda de la incidencia del cáncer de próstata en nuestro medio. Actas Urol Esp. 2001:25 (9):624-627.

26. Unda Urzaiz M, Rodríguez Alcántara F, Badia, García Losa M, Carballido Rodríguez J, Dal-Ré Saavedra R. Características sociosanitarias y diagnósticas del paciente prostático en España a finales del siglo XX. Actas Urol Esp. 2001; 25(3):200-206.

27. Mínguez Martínez R, Fernández Borrell A, Gómez Sancha F, Ruiz Zarate C, Teba del Pino F, Romero Tejada JC, Arellano Gañán R, Pereira Sanz I. Diagnóstico precoz del cáncer de próstata en pacientes con sintomatología prostática mediante TR, PSA, ECOTR y DPSA. Actas Urol Esp 1999;23(8):688-693.

28. Romero Cagigal I, Ferruelo Alonso A, Berenguer Sánchez A. Dieta y cáncer de próstata. Actas Urol Esp. 2003;27(6): 399-409.

29. Ruiz-Ramos M, Escolar Pujolar A. La mortalidad por cáncer de próstata en andalucía: aportaciones al cribado poblacional. Actas Urol Esp. 2005;29(1):41-46.

30. Luján Galán M, Páez Borda A, Romero Cagigal I, Gómez de Vicente JM, Martín Osés E, Berenguer Sánchez A. Papel de la velocidad del PSA en la detección del cáncer de próstata. Un estudio con 986 varones. Actas Urol Esp. 2001; 25(3):193-199.

Dra. S. Granado de la Orden

Servicio de Medicina Preventiva y Gestión de Calidad Hospital General Univ. Gregorio Marañón

Dr. Esquerdo, 46 - 28007 Madrid

E-mail: sgranado.hgugm@salud.madrid.org

(Trabajo recibido el 25 de enero 2006) 